2 Sin DD, Wu L, Anderson JA, et al. Inhaled corticosteroids and mortality in chronic obstructive pulmonary disease. Thorax 2005; 60: 992-997.

3 Weatherall M, Clay J, James K, et al. Dose-response relationship of inhaled corticosteroids and cataracts: a systematic review and meta-analysis. Respirology 2009; 14: 983-990.

4 Weatherall M, James K, Clay J, et al. Dose-response relationship for risk of non-vertebral fracture with inhaled corticosteroids. Clin Exp Allergy 2008; 38: 1451-1458.

5 Hurst JR, Donaldson GC, Quint JK, et al. Temporal clustering of exacerbations in chronic obstructive pulmonary disease. Am J Respir Crit Care Med 2009; 179: 369-374.

6 Pietropaoli AP, Perillo IB, Perkins PT, et al. Smokers have reduced nitric oxide production by conducting airways but normal levels in the alveoli. Inhal Toxicol 2007; 19: 533-541.
7 Tsoukias NM, George SC. A two-compartment model of pulmonary nitric oxide exchange dynamics. J Appl Physiol 1998; 85: 653-666.

8 Sin DD, Lacy P, York E, et al. Effects of fluticasone on systemic markers of inflammation in chronic obstructive pulmonary disease. Am J Respir Crit Care Med 2004; 170: 760-765.

9 Masoli M, Weatherall M, Holt S, et al. Inhaled fluticasone propionate and adrenal effects in adult asthma: systematic review and meta-analysis. Eur Respir J 2006; 28: 960-967.

10 Sin DD, Man SF, Marciniuk DD, et al. The effects of fluticasone with or without salmeterol on systemic biomarkers of inflammation in chronic obstructive pulmonary disease. Am J Respir Crit Care Med 2008; 177: 1207-1214.

\title{
Dose-dependent effects of mandibular protrusion on genioglossus activity in sleep apnoea
}

\section{To the Editors:}

In individuals with a compromised upper airway, the activity of the genioglossus (GG) muscle is augmented to maintain upper airway patency during wakefulness [1]. However, sleep greatly suppresses GG muscle activity, which may lead to partial or complete upper airway collapse in patients with obstructive sleep apnoea (OSA). Mandibular-advancing oral appliances are currently accepted as one promising approach to the treatment of mild-to-moderate OSA [2, 3]. Although enlargement of the upper airway at multiple levels may be important for producing a clinical effect, the contributions of oral appliances to functional aspects, in terms of GG muscle activity, have hardly been documented.

If mandibular advancement increased the electromyographic activity of the GG muscle (GGEMG), this effect should help to maintain the patency of the airway, since the activation of GGEMG could stiffen the compliant airway wall [4]. In contrast, if we consider that mandibular advancement increases the cross-sectional area and decreases the propensity for upper airway occlusion, it would be reasonable to hypothesise that a larger, less collapsible upper airway could improve airflow dynamics, which would require less GGEMG [5]. Accordingly, we investigated the effects of mandibular advancement by an experimental oral appliance on GGEMG and the severity of OSA during sleep.

The study protocol was approved by the Clinical Research Ethics Board at the University of British Columbia (UBC), Vancouver, Canada. A 54-yr-old male patient with severe OSA (body mass index $31.2 \mathrm{~kg} \cdot \mathrm{m}^{-2}$ and apnoea/hypopnoea index (AHI) 30.3 events $\cdot \mathrm{hr}^{-1}$ ) who showed a resolution of symptoms, including daytime sleepiness, snoring and/or apnoea, after complete titration of the oral appliance (Klearway ${ }^{\mathrm{TM}}$; Great Lakes Orthodontics, Tonawanda, NY, USA) was recruited into the study. The patient had used the oral appliance for a period of 4 months prior to the study. The details of the study aims and methods were explained, and written informed consent was obtained.

A custom-made experimental oral appliance was fabricated at the most retruded mandibular position with a vertical opening of the mandible of $2 \mathrm{~mm}$ between the upper and lower incisors. The experimental oral appliance incorporated a pair of custom-made silver/silver chloride ball-type surface electrodes located lingually to record GGEMG [6]. Despite the known significant limitations of surface electrodes compared with needle electrodes, in that the former might pick up geniohyoid and/or mylohyoid muscle activities to some extent [7], we chose to use the surface GG recording technique because it is noninvasive and we were also considering possible future clinical use. A remote-controlled mandibular positioner (RCMP; SagaTech Electronics Inc., Calgary, Canada) was connected to the experimental appliance to reproduce passive forward mandibular movement (see online supplementary material) $[8,9]$. In the first waking session, the patient was asked to insert the experimental oral appliance, to confirm whether the electrodes recorded the GGEMG signal and whether passive mandibular movement with the RCMP was comfortable. Recording was performed from the most retruded mandibular position to the maximum comfortable limit of mandibular advancement with a 1-mm increment forward in the supine body position. Movement of the ribcage was recorded simultaneously. The raw GGEMG signal was monitored with an oscilloscope, in order to confirm a clear and stable inspiratory phasic GGEMG [6]. Thereafter, the patient was scheduled to undergo an overnight polysomnographic sleep study at the sleep laboratory of the UBC Hospital, Vancouver. The same experimental oral appliance with the same recording procedure as in the waking session was used 
in this overnight sleep study. Gradual mandibular advancement using the RCMP was initiated following the onset of stable stage 2 sleep $[8,9]$.

The relationship between the amount of mandibular advancement and the sleeping GGEMG, as well as AHI, was analysed offline by a registered polysomnographic technician at the Neuropsychiatric Research Institute, Tokyo, Japan. All of the electrical signals were converted and stored as a European Data Format file with the use of custom-made software (Resp EMG Analysis; Norupro Light Systems, Tokyo, Japan). In order to compare phasic (maximal activity during inspiration) and tonic (minimal activity during the expiratory phase) GGEMG at each mandibular position, the GGEMG signal was full-wave rectified and averaged for $\geqslant=10$ stable and consecutive respiratory cycles by triggering at the onset of respiratory effort. Thereafter, all of the GGEMG values were standardised to the waking tonic GG activity at $0 \mathrm{~mm}$ mandibular advancement (baseline) and expressed as a percentage of the baseline [10].

After stable breathing was confirmed by the sleep technician, the light was turned off in the room and the patient was asked to sleep. Stage 2 sleep began approximately $<10$ minutes after lights off. Before the patient fell asleep, GGEMG was slightly active, in pace with the inspiratory phase at the $0 \mathrm{~mm}$ protrusive position (fig. 1). In turn, the patient showed a

a)
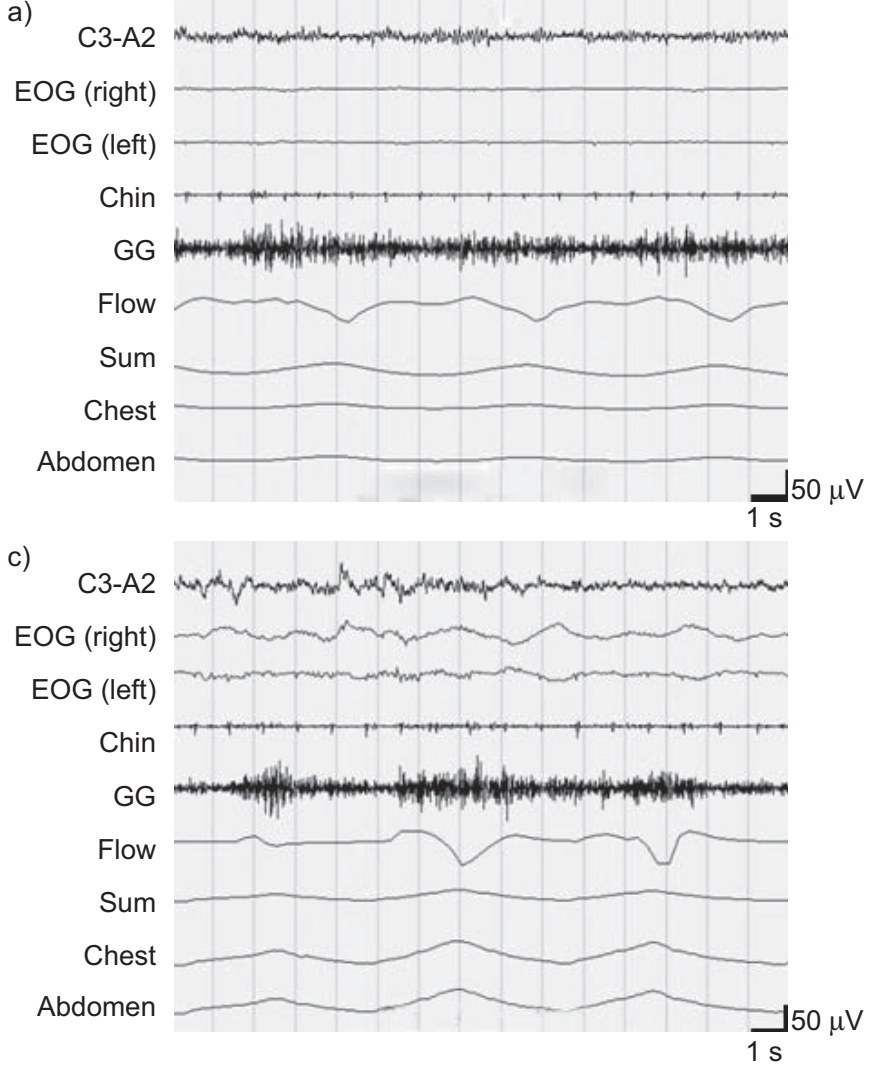

remarkable inspiratory-phase GGEMG at the same mandibular position when stage 2 sleep began.

Both phasic and tonic GGEMG decreased with mandibular protrusion (figs 1 and 2a). Furthermore, the improvement of OSA in terms of AHI was related to the amount of mandibular advancement: $0-4 \mathrm{~mm}$ advancement did not eliminate OSA (fig. 2b). However, $8 \mathrm{~mm}$ mandibular protrusion, which was the maximal comfort limit of mandibular protrusion in this patient, eliminated both apnoeas and hypopnoeas.

In the total sleep time of the RCMP study (70 $\mathrm{min})$, stage 2 lasted for $52 \mathrm{~min}$ (74.3\% of the study time). Protrusion of the mandible did not induce arousal throughout the study. The duration of AHI measurement at each mandibular position was: $44 \mathrm{~min}$ at $0 \mathrm{~mm} ; 5.5 \mathrm{~min}$ at $2 \mathrm{~mm} ; 4.5 \mathrm{~min}$ at $4 \mathrm{~mm}$; $4.0 \mathrm{~min}$ at $6 \mathrm{~mm}$; and $5.0 \mathrm{~min}$ at $8 \mathrm{~mm}$.

This is the first study to demonstrate the dose-dependent effects of mandibular advancement on GGEMG in OSA during sleep. Our results suggest that an oral appliance acts directly to enlarge the airway size without the need for an increase in GGEMG. Furthermore, we conjectured that enlargement of the airway by an oral appliance enabled the GG muscle to normalise its hyperactivity, which is commonly present in OSA patients.

The degree of mandibular protrusion is a key variable that affects oral appliance efficacy, since the advancement b)

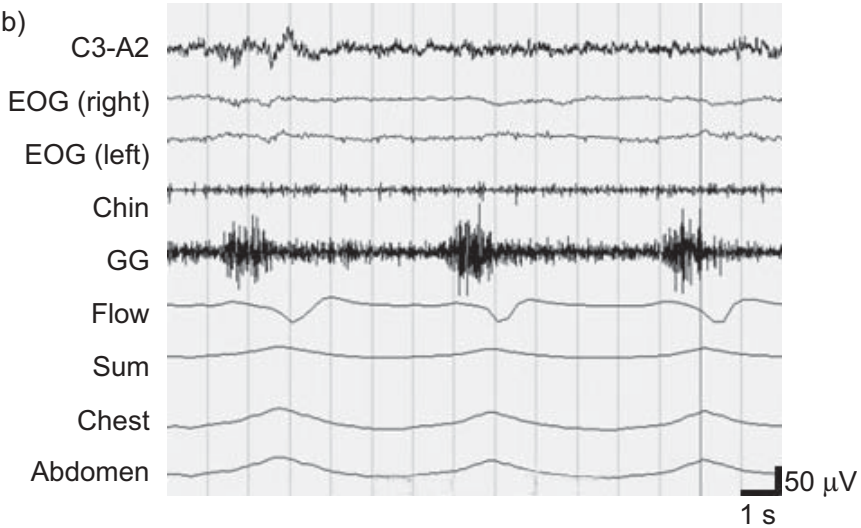

d)

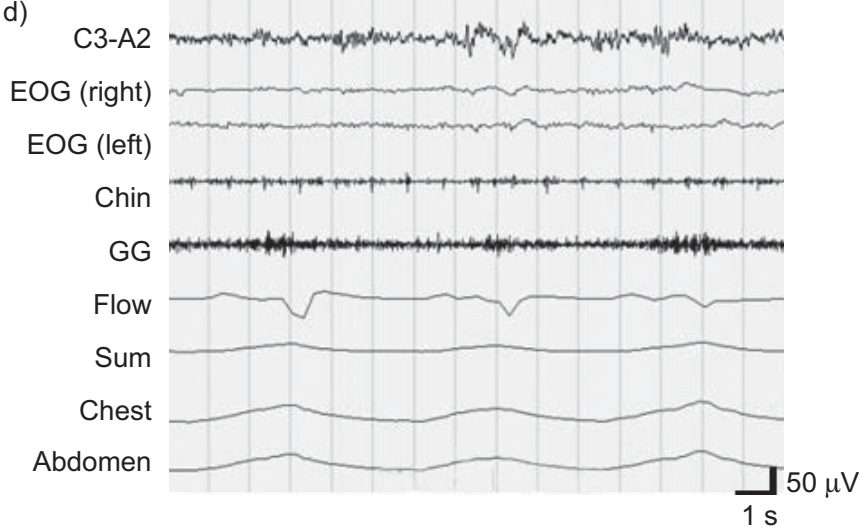

FIGURE 1. Dose-dependent decreases in genioglossus (GG) muscle activity in association with remote-controlled mandibular advancement during sleep. The GG trace represents raw GG muscle activity that was recorded instead of leg muscle activity. a) Waking, 0 mm protrusion; b) stage 2 sleep, 0 mm protrusion; c) stage 2 sleep, 4 mm protrusion; d) stage 2 sleep, 8 mm protrusion. EOG: electro-oculography. 

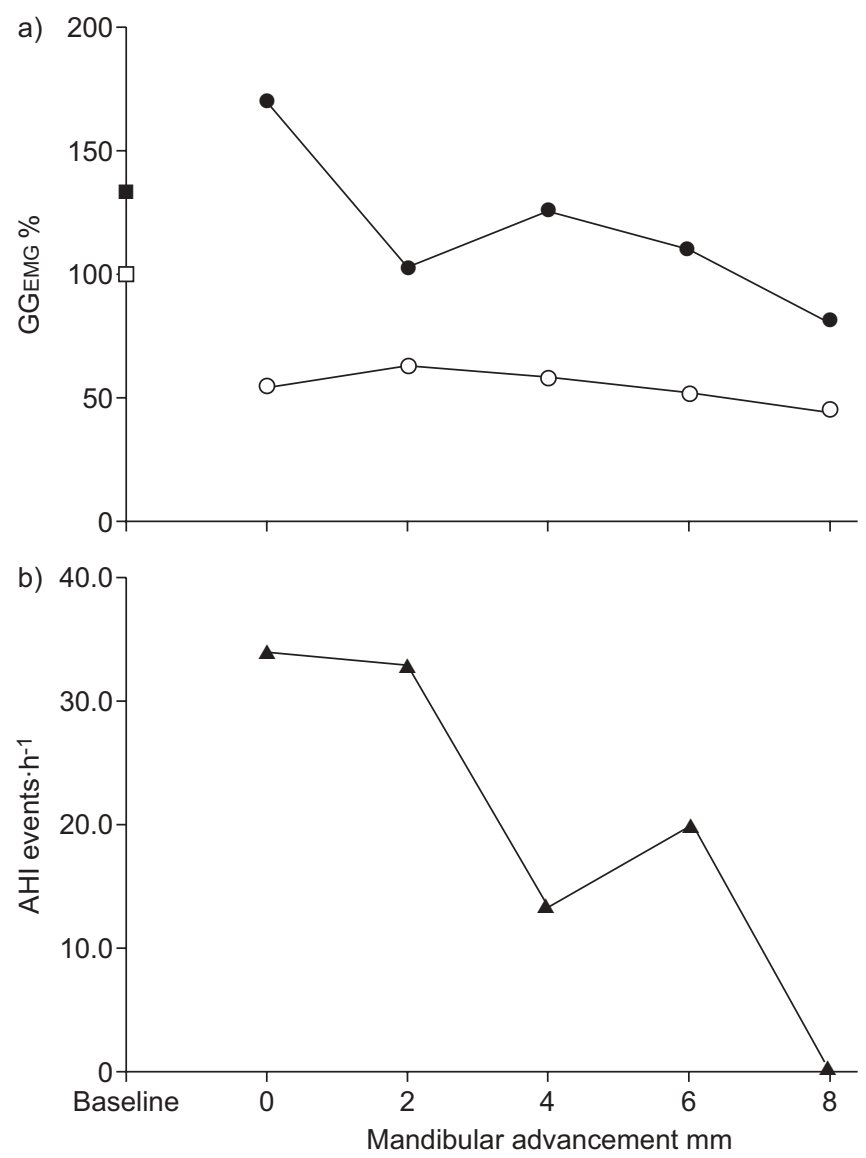

FIGURE 2. Relationship between the amount of mandibular advancement, and a) genioglossus muscle activity (GGEMG) ( $\bigcirc$ : tonic; $\bullet$ : phasic) and b) apnoea/ hypopnoea index (AHI). Note that all of the GGEMG values were standardised to the waking tonic GGEMG at $0 \mathrm{~mm}$ mandibular advancement (baseline; $\square$ ) and expressed as a percentage of baseline. The waking phasic GG activity at $0 \mathrm{~mm}$ mandibular advancement is also shown (

manoeuvre enlarges the constricted upper airway [2,3]. Interestingly, the reduction in GGEMG in this study was similar to the effect of nasal continuous positive airway pressure therapy, in that successful application of continuous positive airway pressure decreased GGEMG in OSA patients [11], although the approach used to improve airway patency was different between the two treatment modalities. OLIVEN et al. [12] previously demonstrated the smaller contribution of anatomy to the prevalence of OSA and speculated that the level of neuromuscular activity might ultimately determine the occurrence of OSA. However, our results suggested that the therapeutic outcomes of oral appliance therapy for OSA are likely to be anatomical [13] rather than via the activation of upper airway muscle tone.

With the use of an endoscopic technique, we previously demonstrated that the cross-sectional area of the upper airway at end-tidal expiration was maintained during inspiration despite a more negative intraluminal pressure, possibly because of the augmentation of GGEMG in an effort to protect upper airway patency in waking OSA patients [5]. We also found that the cross-sectional area became significantly smaller during inspiration despite mandibular advancement, although the cross-sectional area at end-tidal expiration increased, as expected. We speculated that input from upper airway mechanoreceptors during inspiration decreased phasic GGEMG, since protrusion of the mandible reduced the propensity for upper-airway collapse [5]. Based on the results of the present study and the previous report [5], we can further hypothesise that GGEMG activity could be controlled to maintain a target level of cross-sectional area and patency of the upper airway. We recognise that this study merely demonstrates associations, not causality. The sleep session was performed only during stage 2 sleep, as in a previous report $[8,9]$ and the changes in the GGEMG response in other stages of sleep [10] also need to be tested. To avoid inducing arousal by the RCMP machine, we recorded GGEMG activity during such shorter duration (4.4-5.5 $\mathrm{min}$ ) at each mandibular position and, therefore, had to evaluate AHI using data obtained during that period. The results also need to be confirmed in a larger patient sample with consideration of recording time. Also, surface electromyography data should be compared with needle-electrode signals in a future RCMP study.

In conclusion, incremental advancement of the mandible in one OSA patient reduced GGEMG during sleep.

\section{F.R. Almeida*, S. Tsuiki ${ }^{\#,}$, Y. Hattori ${ }^{+}$, Y. Takei ${ }^{\#}$, Y. Inoue I, $^{\#}$ and A.A. Lowe}

*Dept of Oral, Biological and Medical Sciences, and ${ }^{\S}$ Division of Orthodontics, Dept of Oral Health Sciences, Faculty of Dentistry, The University of British Columbia, Vancouver, Canada. "Japan Somnology Center, Neuropsychiatric Research Institute, "Dept of Somnology, Tokyo Medical University, Tokyo, and ${ }^{+}$Division of Aging and Geriatric Dentistry, Dept of Oral Function and Morphology, Tohoku University Graduate School of Dentistry, Sendai, Japan.

Correspondence: S. Tsuiki, Japan Somnology Center, Neuropsychiatric Research Institute, 1-24-10, Yoyogi, Shibuya-ku, Tokyo 151-0053, Japan. E-mail: tsuiki@somnology.com

Support Statement: This study was supported by the British Columbia Health Research Foundation and Grants-in-Aid for Scientific Research Projects (20592422 and 21406033) from the Japan Society for the Promotion of Science.

Statement of Interest: Statements of interest for F.R. Almeida, S. Tsuiki and A.A. Lowe can be found at www.erj.ersjounals. $\mathrm{com} / \mathrm{site} / \mathrm{misc} /$ statements.xhtml

Acknowledgements: The authors would like to thank SagaTech Electronics Inc., Calgary, Canada for providing the RCMP prototype for this study and I. Ellis (Faculty of Dentisty, The University of British Columbia, Vancouver, Canada) for her expertise in editorial assistance.

\section{REFERENCES}

1 Sériès F. Upper airway muscles awake and asleep. Sleep Med Rev 2002; 6: 229-242. 
2 Ferguson KA, Cartwright R, Rogers R, et al. Oral appliances for snoring and obstructive sleep apnea: a review. Sleep 2006; 29: 244-262.

3 Tsuiki S, Kobayashi M, Namba K, et al. Optimal positive airway pressure predicts oral appliance treatment response to sleep apnoea. Eur Respir J 2010; 35: 1098-1105.

4 Isono S. Upper airway muscle function during sleep. In: Loughlin GM, Varroll JL, Marcus CI, eds. Sleep and Breathing in Children. New York, Marcel Dekker, Inc., 2000; pp. 261-291.

5 Tsuiki S, Ryan CF, Lowe AA, et al. Functional contribution of mandibular advancement to awake upper airway patency in obstructive sleep apnea. Sleep Breath 2007; 11: 245-251.

6 Adachi S, Lowe AA, Tsuchiya M, et al. Genioglossus muscle activity and inspiratory timing in obstructive sleep apnea. Am J Orthod Dentofac Orthop 1993; 104: 138-145.

7 Johal A, Gill G, Ferman A, et al. The effect of mandibular advancement appliances on awake upper airway and muscle activity in patients with obstructive sleep apnoea. Clin Physiol Funct Imaging 2007; 27: 47-53.
8 Tsai WH, Vazquetz JC, Oshima T, et al. Remotely controlled mandibular positioner predicts efficacy of oral appliances in sleep apnea. Am J Respir Crit Care Med 2004; 170: 366-370.

9 Dort LC, Hadjuk E, Remmers JE. Mandibular advancement and obstructive sleep apnoea: a method for determining effective mandibular protrusion. Eur Respir J 2006; 27: 1003-1009.

10 Jordan AS, White DP, Lo YL, et al. Airway dilator muscle activity and lung volume during stable breathing in obstructive sleep apnea. Sleep 2009; 32: 361-368.

11 Strohl KP, Redline S. Nasal CPAP therapy, upper airway muscle activation, and obstructive sleep apnea. Am Rev Respir Dis 1986; 134: 555-558.

12 Oliven A, Aspandiarov E, Gankin I, et al. Collapsibility of the relaxed pharynx and risk of sleep apnoea. Eur Respir J 2008; 32: 1309-1315.

13 Kairaitis K, Stavrinou R, Wheatley JR, et al. Mandibular advancement decreases pressures in the tissues surrounding the upper airway in rabbits. J Appl Physiol 2006; 100: 349-356.

\section{Detection of right ventricular dysfunction by tissue Doppler imaging in asymptomatic patients with pulmonary sarcoidosis}

\section{To the Editors:}

Tissue Doppler imaging (TDI) is a relatively new ultrasound modality in echocardiography, which is used to detect left and right ventricular functional abnormalities early and accurately by recording systolic and diastolic velocities of the mitral and tricuspidal annulus, respectively. The value of this method has been corroborated on numerous studies describing right ventricular (RV) dysfunction in a variety of systemic diseases with pulmonary and/or cardiovascular involvement [1].

Sarcoidosis is a multisystem granulomatous disease of unknown aetiology characterised by cardiorespiratory manifestations, among others. RV dysfunction is often apparent but not clinically recognised until pulmonary hypertension has been developed [2]. The purpose of this study was to evaluate RV function in patients with sarcoidosis by the use of ultrasound, including the TDI modality, and correlate it with clinical, respiratory and cardiac parameters.

We conducted an observational case-control study. Consecutive sarcoidosis patients were recruited from the outpatient Sarcoidosis Clinic of the General Hospital of Chest Diseases of Athens, Athens, Greece between October 2007 and June 2008. The primary criterion for enrolment was the presence of biopsyproven pulmonary sarcoidosis without the presence of cardiac involvement, according to the modified criteria of the Japanese Ministry of Health and Welfare [3]. The exclusion criterion was the presence of any associated disease that could influence systolic and/or diastolic properties of the heart. Subgroup analyses were performed with the patients divided in two groups based on the therapy administered: a subgroup with patients who did not receive any therapy and a subgroup with patients who received any kind of therapy (cortisone, etc.). Those who did not receive any medication were further classified into groups according to the disease stage at which the patients originally presented. All patients were compared to healthy volunteers. The two groups (patients and healthy controls) were age-, sex- and body mass index-matched. None of the patients or control subjects was receiving any cardiac medication. Data regarding the clinical evaluation of disease severity, including symptoms (including functional ability according to the New York Heart Association (NYHA) scale), chest radiograph, high-resolution computed tomography imaging and lung function tests (including forced expiratory volume in $1 \mathrm{~s}$ (FEV1), forced expiratory vital capacity (FVC), FEV1/FVC, total lung capacity and diffusing capacity of the lung for carbon monoxide $(D \mathrm{~L}, \mathrm{CO}))$ were collected. Radiographic staging was estimated using conventional chest radiography [4]. Echocardiography was performed with a commercially available ultrasonic device (Sonos 5500; HewlettPackard, Andover, MA, USA). Two-dimensional measurements, and conventional Doppler and TDI recordings of both the free-wall side of the mitral and tricuspid annulus were acquired [5]. This study was approved by the Institutional Ethical Committee (General Hospital of Chest Diseases "Sotiria", Athens, Greece) and informed consent was obtained from all participants.

Distribution normality of the continuous variables was assessed by the Kolmogorov-Smirnov test. Differences among different subgroups were evaluated by one-way ANOVA, with Scheffe post hoc analysis for multiple comparisons. Bivariate 